\title{
Introducción al problema inverso electrocardiográfico
}

\author{
Esteban Herrera Hernandez ${ }^{1}$, Rafael Lemuz López ${ }^{1}$, Carlos Guillén Galván \\ Ángel Ramos del Olmo ${ }^{2}$ \\ ${ }^{1}$ Benemérita Universidad Autónoma de Puebla, Facultad de Ciencias de la Computación, \\ Puebla, México \\ ${ }^{2}$, Universidad Complutense de Madrid, Departamento de Matemática Aplicada \\ Madrid, España \\ artanis_ii@hotmail.com,rlemuz@cs.buap.mx,cguillen@fcfm.buap.mx,angel@mat.ucm.es
}

Resumen. En este trabajo se presenta una propuesta de adquisición de datos para el problema inverso en electrocardiografía utilizando un sistema de adquisición inalámbrico de bajo costo. Obtener una imagen de la actividad eléctrica en la superficie del corazón resolviendo el problema inverso es importante en el estudio y diagnóstico de enfermedades cardiacas. Además, se presenta una introducción al planteamiento matemático del problema describiendo dos métodos de regularización: el método de Tikhonov y la Descomposición de Valores Singulares Truncados (TSVD).

Palabras clave: Adquisición de datos, ecuaciones diferenciales parciales, problema inverso, electrocardiograma (ECG), EPOC headset.

\section{Introduction to the Inverse Electrocardiographic Problem}

\begin{abstract}
In this work, a data acquisition approach for the inverse problem in electrocardiography using a low cost wireless acquisition system is presented. Obtaining an image of the electrical activity on the heart surface is important in the study and diagnosis of heart disease. In addition, an introduction to the mathematical problem is presented describing two regularization methods: the Tikhonov method and the Truncated Singular Values Decomposition (TSVD).
\end{abstract}

Key words: Data acquisition, partial differential equations, inverse problem, electrocardiogram (ECG), EPOC headset. 


\section{Introducción}

Las enfermedades cardiovasculares (ECV) son la principal causa de muerte en todo el mundo. Cada año mueren más personas por ECV que por cualquier otra causa. En México es el segundo lugar como causa de muerte, durante el año 2014 se registraron 82,334 decesos por enfermedades isquémicas del corazón lo cual es preocupante [4], ya que por nuestro ritmo de vida esto se incrementa año con año y muchos de los estudios que existen para diagnosticar este tipo de enfermedades son excesivamente caros al igual que sus tratamientos por lo que, resolviendo el problema inverso electrocardiográfico podría tener un impacto muy fuerte en la prevención de enfermedades cardiacas.

El problema inverso electrocardiográfico consiste en obtener potenciales miocárdicos a partir de los registros de superficie de manera no invasiva. Los registros superficiales se obtienen con la técnica de BSPM (Body Suface Potential Mapping) con alrededor de 32 a 300 electrodos alrededor del torso. Con ello se pretende encontrar regiones del corazón donde están ocurriendo patologías cardiacas, como son el bloqueo de rama, insuficiencia cardiaca, infarto del miocardio, potenciales tardíos ventriculares o el síndrome de Brugada, algunas de ellas causantes de la denominada muerte súbita.

En la literatura se dice que el problema inverso electrocardiográfico está mal planteado resultando en múltiples soluciones posibles e inestabilidad. Por tanto, es necesario el desarrollo de técnicas y algoritmos nuevos que simplifiquen y permitan aplicar estos resultados a una mayor cantidad de población.

En condiciones normales, existe una señal de naturaleza eléctrica que recorre el corazón de forma periódica y secuencialmente ordenada, que permite un bombeo eficaz de la sangre al resto de organismos del cuerpo a través del sistema circulatorio.

El corazón es una parte importante de este sistema, y suministra todos los demás órganos con sangre oxigenada. Este órgano consiste en cuatro cámaras, la aurícula izquierda y la derecha y el ventrículo izquierdo y derecho. La contracción, generada por un estímulo, provoca el bombeo de sangre a través de la circulación.

Este estímulo surge en una región llamada el nódulo sinusal situado en la aurícula derecha. El impulso llega a las células musculares por el sistema de conducción del corazón. La corriente eléctrica resultante controla la contracción del corazón y se puede medir en la superficie del cuerpo. Si esta corriente eléctrica se ve alterada puede provocar una arritmia.

\section{Métodos de medición}

Existen dos métodos para medir el potencial eléctrico, unipolar y bipolar. Para medir un potencial eléctrico, es necesario utilizar al menos dos electrodos. En la medición unipolar, un electrodo se coloca cerca del corazón para medir la señal y el otro electrodo se coloca lejos del corazón como referencia. En la medición bipolar, dos electrodos tienen aproximadamente la misma distancia desde el corazón.

El estándar de 12 derivaciones de ECG, es un sistema que incluye tanto conductores bipolares como unipolares y es estándar en la práctica clínica actual. En este caso diez electrodos se colocan en el cuerpo y 2 son referencias. En ellas se registran doce señales eléctricas diferentes en aproximadamente al mismo tiempo [2]. Mientras más 
electrodos superficiales se utilicen mejores resultados se obtendrán. Los electrodos se colocan directamente sobre la superficie del cuerpo y son capaces de tomar registros sobre la actividad eléctrica. Los materiales de los que se constituyen la mayoría de los electrodos son muy variables, entre ellos están la plata, el oro, el acero inoxidable, platino, entre otros [3].

\section{Problema inverso}

Para poder obtener datos y resolver el problema inverso en electrocardiografía, tenemos que interpretar los potenciales registrados en la superficie del cuerpo debido a la actividad eléctrica del corazón. El objetivo general de los problemas "inversos" llamados "hacia adelante" de electrocardiografía es una mejor comprensión cualitativa y cuantitativa de la actividad eléctrica del corazón [1].

Para poder medir la actividad eléctrica en la superficie del corazón utilizaremos un sistema de adquisición de bajo costo el cual es el EPOC Headset, ya que este sistema cuenta con sensores de humedad a base de solución salina (sin geles pegajosos) y 14 canales de EEG, más 2 canales de referencia y nos ofrece un posicionamiento óptimo para la adquisición de señales, es inalámbrico y recargable.

Este sistema nos ayudara a obtener la información necesaria sobre las señales P,T y el complejo QRS que mencionamos anteriormente, a través de electrodos superficiales puestos en 4 partes del torso, de los cuales 2, estarán cerca del brazo derecho y del brazo izquierdo, otro electrodo irá junto a la pierna izquierda y por último el electrodo que va a estar junto a la pierna derecha será nuestra referencia, el resto de los electrodos se colocarán para medir la actividad eléctrica de los ventrículos ver figura 1 . Con esto se podremos obtener los datos necesarios para poder resolver el problema inverso mediante ecuaciones parciales y la descomposición de valores singulares regularizados y así obtener el mapeo de la actividad eléctrica en la superficie del corazón.
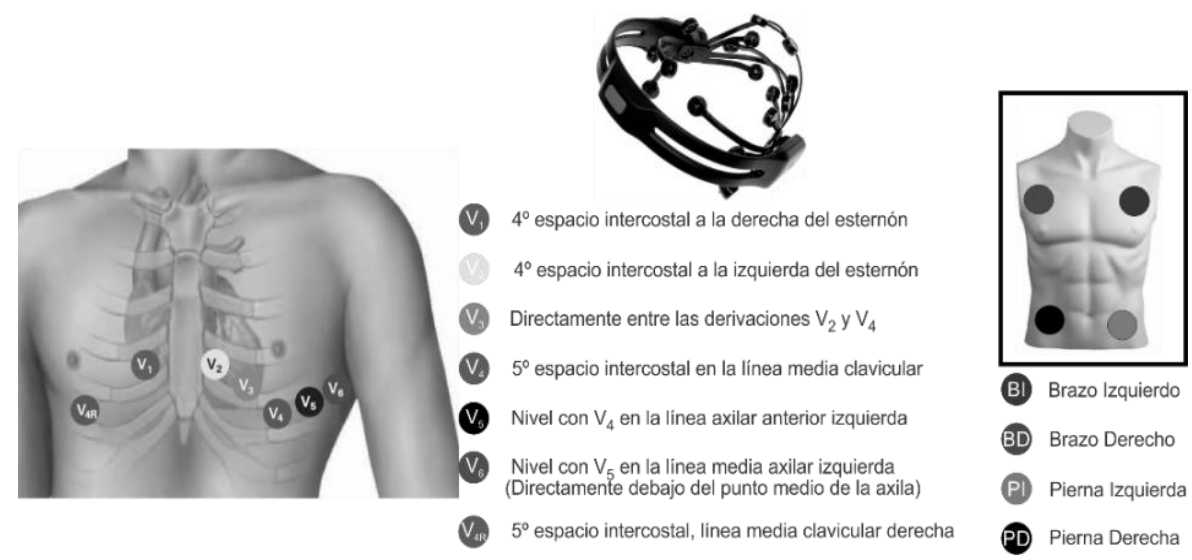

Fig. 1. 12 Derivaciones de ECG y EPOC Headset.

Se sabe que cuando existe alguna alteración en las señales $\mathrm{P}, \mathrm{T}$ y el complejo QRS, el paciente puede sufrir de alguna enfermedad del corazón. En la figura 2 se muestra 
los signos vitales de un corazón con ritmo sinusal, taquicardia ventricular, fibrilación auricular y fibrilación ventricular.

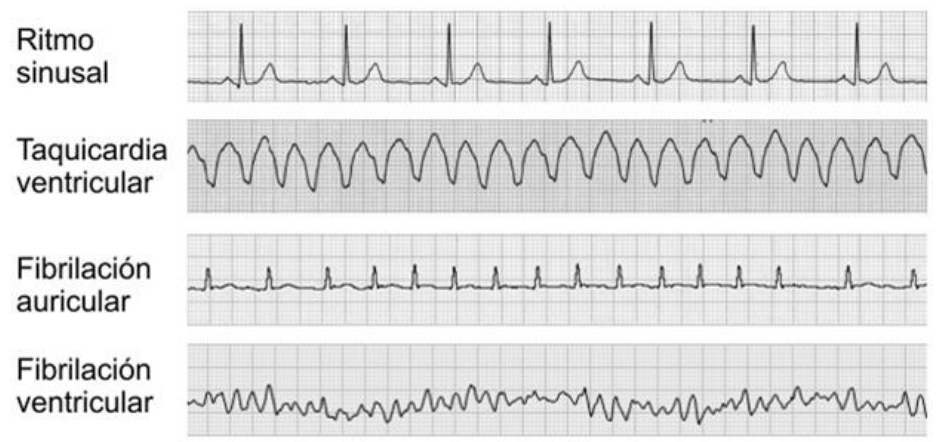

Fig. 2. Enfermedades del corazón.

\section{Métodos matemáticos}

Para comprender el enfoque matemático del problema inverso de electrocardiografía se requiere un conocimiento básico. Aquí explicaremos algunas definiciones y teoremas, que se necesitan saber para manejar un problema mal planteado.

Definición: Un modelo matemático es un mapeo

$$
A: X \rightarrow Y .
$$

Del conjunto de causas (parámetros) $X$ para el conjunto de la dirección (datos) $Y$. En el problema directo el efecto se calcula en función de la causa, es decir, para $x \in$ $X$ calculamos $A x \in Y$. A la inversa, en el problema inverso tenemos que observar $y \in Y$ y tratar de encontrar una $x \in X$ cuando $A x=y$.

La siguiente definición debido a Hadamard es una clasificación de los problemas bien planteados y mal planteados.

Definición: Sea $A: X \rightarrow Y$ un mapeo entre espacios topológicos $X$ y $Y$. El problema $(A, X, Y)$ se llama bien planteado, si las siguientes condiciones son cumplidas:

a) Para toda $y \in Y$ la ecuación $A x=y$ es solución de $x \in X$.

b) La solución es única.

c) La aplicación inversa $A^{-1}: Y \rightarrow X$ es continua, es decir la solución de $x$ depende de forma continua de los datos en $y$ (pequeños errores en $y$ harán pequeños errores en $x$ ). 


\subsection{Inversa generalizada}

Las dificultadesk en la resolución de un problema mal planteado son obvias. No podemos asumir que una solución es única, o que siquiera existe. Por lo tanto, tenemos que encontrar una manera de satisfacer las condiciones a) y c).

En lo que sigue, suponemos que $X$ y $Y$ sean espacios de Hilbert y $A$ para ser un operador lineal, $A \in L(X, Y)$. Analizamos la solución de la ecuación [10]:

$$
A x=y .
$$

Con un determinado arbitrario $y \in Y$. Si el rango de $A: R(A)$ no es el espacio completo de la imagen, no es solucionable para $y \in Y$. Por otro lado, si $A$ tiene un espacio nulo trivial $N(A)$, la solución no es única. Así que si hay varias soluciones, es razonable elegir el que tenga la norma mínima entre ellos. Este pensamiento lleva a la siguiente definición:

Un elemento $x \in X$ es llamado:

a) Mínimos cuadrados solución de la ecuación $A x=y$ si

$$
\|A x-y\|_{Y}=\inf \left\{\|A z-y\|_{Y}\right\} z \in X .
$$

b) Solución de norma mínima de la ecuación $A x=y$ si

$\|x\|_{X}=\inf \left\{|| z||_{X} \mid z\right.$ es una solución de mínimos cuadrados de $\left.A x=y\right\}$.

Todavía no podemos asegurar que existe una solución de mínimos cuadrados para una y arbitraria, ya que $R(A)$ no necesita ser cerrado. Pero por ahora, esto se puede suponer. En este caso, la solución de norma mínima es única, debido a la convexidad de la minimización relativa funcional. A continuación, se define la inversa generalizada Moore-Penrose, con la que podemos calcular la solución de la norma mínima [2].

Definición: Sea $A$ un continuo funcional lineal entre espacios de Hilbert $X$ y $Y$, es decir, $a \in L(X, Y)$. Sea $A: N(A)^{\perp} \rightarrow R(A)$ sea su restricción. A continuación, la inversa generalizada de Moore-Penrose $A^{\dagger}$ se define como la extensión lineal única de $A^{-1}$ a su dominio

$$
D\left(A^{\dagger}\right):=R(A) \oplus R(A)^{\perp}
$$

Con

$$
N\left(A^{\dagger}\right)=R(A)^{\perp} \text {. Así }\left(A^{\dagger}\right) \text { son mapas } D\left(A^{\dagger}\right) \text { para } N(A)^{\perp} .
$$

Como se ha mencionado antes, la inversa generalizada se puede utilizar para calcular la solución mínima. Esto lo podemos ver en el siguiente teorema.

Teorema: La ecuación $A x=y$ tiene una solución única por la norma-mínima dada por

$$
x^{\dagger}:=A^{\dagger} y \quad \forall y \in D\left(A^{\dagger}\right)
$$

El conjunto de todas las soluciones de mínimos cuadrados está dada por $\left\{x^{\dagger}\right\}+$ $N(A)$. Con este Teorema, podemos encontrar una solución única para la ecuación $A x=y$ si $y \in D(A)$. Por lo tanto, las condiciones a) y b) de la definición anterior son 
cumplidas. Si $R(A)$ no está cerrado y por lo tanto no existe $y \in Y \backslash D\left(A^{\dagger}\right)$, porque se viola la tercera condición. Hay algunas técnicas de regularización, que ayudan a manejar este caso [2].

\section{Técnicas de regularización}

Como ya se ha mencionado, el problema inverso no posee una solución única y como hemos visto el problema inverso está mal planteado, es decir, el operador $R^{-1}$ no tiene límites, por lo tanto, los pequeños errores en los datos medidos $d$ pueden ser críticos e introducir grandes errores en $g$. Por esa razón, tenemos que encontrar una manera de manejar esta dificultad.

Hay algunas técnicas de regularización, que se aproximan al problema mal planteado mediante el uso de ecuaciones bien planteadas. A continuación, se presentan la Regularización de Tikhonov y la descomposición de Valores Singulares Truncados (TSVD) [10].

\subsection{Regularización de Tikhonov}

Este método de regularización se inspira en la formulación discreta de mínimos cuadrados [10]. Por esta razón, se introduce la función

$$
J(g)=\|R(g)-d\|_{L^{2}\left(\sigma_{1}\right)}^{2} \text { para } g \in H^{1}\left(\sigma_{3}\right),
$$

en la que usamos la misma denotación como antes.

Ahora volvemos a escribir la ecuación $R(g)=d$ como el problema de minimización

$$
\min _{g \in H^{1}\left(\sigma_{3}\right)} J(g) .
$$

Por supuesto, mediante la formulación del problema inverso de esta forma, el "mal planteado" aún está presente. Con el siguiente paso, nos aproximamos a (8) por un problema de buen comportamiento. Se define una nueva función $j \in(\mathrm{g})$ mediante la adición de un término de regularización a $j(\mathrm{~g})$ :

$$
\begin{gathered}
J_{\epsilon}(g)=J(g)+\epsilon\|g\|_{L^{2}\left(\sigma_{3}\right)}^{2}=\|R(g)-d\|_{L^{2}\left(\sigma_{1}\right)}^{2}+\epsilon\|g\|_{L^{2}\left(\sigma_{3}\right)}^{2} \\
\text { para } g \in H^{1}\left(\sigma_{3}\right),
\end{gathered}
$$

con $\epsilon>0$ como el parámetro de regularización. Con (9) se formula una aproximación de (8):

$$
\min _{g \in H^{1}\left(\sigma_{3}\right)} J(g) .
$$

Esto es un compromiso entre minimizar la norma de la residual y mantener $g$ pequeña. Por último, se demuestra que la adición del término de regularización a nuestra función original hace cumplir la estabilidad.

Recordamos que $g \in H^{1}\left(\sigma_{3}\right)$ se puede escribir en la forma 


$$
g(x)=\sum_{K=0}^{\infty} p k \cos (k \pi x) \text { para la adecuada } p 0, p 1, \ldots \in \mathbb{R} .
$$

Por otra parte con $J_{\epsilon}=J_{\epsilon}(p 0, p 1, \ldots)$.

$\mathrm{Y}$ desde el primer orden la condición necesaria

$$
\frac{\partial J_{\varepsilon}}{\partial P_{i}}=0 \text { para } i=1,2, \ldots
$$

Durante un mínimo, se deriva la formula

$$
p k=\frac{d_{K} \cosh (k \pi)}{1+\epsilon \cosh ^{2}(k \pi)}
$$

para los coeficientes de Fourier de la solución $g$ de (10), véase [9]. Por lo tanto, podemos concluir, que

$$
g(x)=\sum_{K=0}^{\infty} \frac{d_{K} \cosh (k \pi)}{1+\epsilon \cosh ^{2}(k \pi)} \cos (k \pi x)
$$

Resuelve (10):

$$
\begin{aligned}
& d_{k}=2 \int_{\epsilon} d(x) \cos (k \pi x) d x, \text { para } k=1,2, \ldots, \\
& d_{0}=\int_{\epsilon} d(x) d x .
\end{aligned}
$$

siendo los coeficientes de Fourier los datos de medición $d$. Ahora obtenemos la siguiente aproximación $R_{\epsilon}^{-1}$ del operador inverso $R^{-1}$ del mapeo de $R$ :

$$
R_{\epsilon}^{-1}(d)=R_{\epsilon}^{-1}\left(\sum_{K=0}^{\infty} d_{K} \cosh (k \pi x)\right)=\sum_{K=0}^{\infty} d_{K} \frac{\cosh (k \pi)}{1+\epsilon \cosh ^{2}(k \pi)} \cos (k \pi x) .
$$

Y con

$$
\lambda_{K}=\frac{1}{\cos (k \pi)} \text { para } k=0,1, \ldots
$$

Siendo los valores propios de R, obtenemos:

$$
R_{\epsilon}^{-1}=\sum_{K=0}^{\infty} d_{K} \frac{\lambda_{k}}{\lambda_{k}^{2}+\epsilon} \cos (k \pi x)
$$

Si comparamos $R^{-1}$ y el $R_{\epsilon}^{-1}$ aproximado, vemos que, por una parte, para los componentes de baja frecuencia de $d$, la acción de $R^{-1}$ y $R_{\epsilon}^{-1}$ son casi idénticos. Por otra parte, los componentes de alta frecuencia de $d$ se amortiguan de manera eficiente por $R_{\epsilon}^{-1}$.Además de eso, podemos concluir, que los pares de valores propios de la función propia del $R_{\epsilon}^{-1}$ son: 


$$
\lambda_{\epsilon, k}^{-1}=\frac{\cosh (k \pi)}{1+\epsilon \cosh ^{2}(k \pi)}, \epsilon_{\epsilon, k}=\cos (k \pi x) \text { para } k=0,1, \ldots
$$

Mediante el uso de este, se muestra que $R_{\epsilon}^{-1}$ es un operador lineal acotado para cada $\epsilon>0$ es fijo. Vemos que para $k=0,1, \ldots$

$$
\begin{aligned}
& \lambda_{\epsilon, k}^{-1}=\frac{\cosh (k \pi x)}{1+\epsilon \cosh ^{2}(k \pi)} \leq \frac{\epsilon^{-1}\left(1+\epsilon \cosh ^{2}(k \pi)\right)}{\left(1+\epsilon \cosh ^{2}(k \pi)\right)}=\frac{1}{\epsilon^{\prime}} \\
& \lambda_{\epsilon, k}^{-1}>0 .
\end{aligned}
$$

Y de eso, podemos concluir

$$
\lambda_{\epsilon, k} \geq \epsilon>0 .
$$

En consecuencia, el cero no es un punto adherente de $\lambda_{\epsilon}$ valores propios de $R_{\epsilon}$. El resultante $R_{\epsilon}^{-1}$ es entonces un operador lineal acotado y por lo tanto continua, a condición de que $\epsilon>0$.De esta manera, el problema (10) está bien planteado para cada $\epsilon>0$.Todavía hay un reto en encontrar el equilibrio adecuado entre el tamaño de $\epsilon \mathrm{y}$ el orden de los errores presentes en $d$. Nosotros no nos extenderemos sobre este tema. Un ejemplo referente a esta materia se puede encontrar en [9].

\subsection{Descomposición de valores singulares truncados}

Otro método de regularización es la descomposición de valores singulares truncados (TSVD). La idea de esta técnica es la obtención de un nuevo problema con una matriz de transferencia deficiente con un rango bien acondicionado.

\section{Matriz de transferencia}

Sea $R$ el mapeo se define en $R: H^{1}\left(\sigma_{3}\right) \rightarrow L^{2}\left(\sigma_{1}\right)$ y $R(g)=u(g) \mid \sigma_{1}$. También podemos decir que $R$ es una función de transferencia entre la fuente y el $g$ desconocido (ruido dañado) de superficie corporal potencial $d$. Es posible construir una matriz de transferencia explícita $A$ de $g$ a $d$, es decir,

$$
A g=d
$$

Ahora sólo discutiremos algunas propiedades de $A$ y las utilizaremos para resolver el problema inverso.

\section{Descomposición de valores singulares}

Antes de pasar a TSVD, es necesario entender la teoría detrás de este método de regularización. La Descomposición en valores singulares (SVD) es una técnica para factorizar una matriz para determinar los componentes principales de la información que contiene.

Si utilizamos un SVD de la matriz de transferencia A, obtenemos la siguiente fórmula:

$$
A=U \Sigma V^{T},
$$


con $U$ y $V$ de ser matrices ortogonales y $\Sigma$, una matriz diagonal, que contienen los valores singulares. Es obvio que:

$$
A^{T}=U \Sigma V^{T} .
$$

es un SVD para $A^{T}$. También podemos repetir la fórmula (13):

$$
A \sum_{i=1}^{N} \sigma_{i} u_{i} v_{i}^{t}
$$

cuando $\sigma_{i}$, son los valores singulares para $i=1, \ldots, N$ y $u_{i}$ y $v_{i}$ son vectores que forman las matrices ortogonales. Por lo tanto, $A$ y $A^{T}$ tienen los mismos valores singulares. Por lo general, los valores singulares son ordenados en orden decreciente de tamaño y si $\sigma_{1} \geq \sigma_{2} \geq \ldots \sigma_{r}>\sigma_{r}+1=\ldots=\sigma_{N}=0$, podemos concluir que $R(A)=r$.

Ahora describiremos los vectores $u_{i}$ y $v_{i}$ con más detalle. Ellos pueden ser separados en dos grupos. Los vectores $u_{i} \mathrm{y} v_{i}$, que corresponden a pequeños valores singulares, a menudo se descartan, porque se supone otorgar al espacio ruido aleatorio. Los vectores $u_{i}$ y $v_{i}$ que permanecen, son los principales componentes y también se conoce como los vectores propios. Determinamos, que valores singulares corresponden al espacio de ruido por el trazado de los valores singulares ordenados contra el rango de la matriz obtenida a partir del subconjunto apropiado de esos valores singulares.

\section{Descomposición de valores singulares truncados (TSVD)}

Ahora pasamos a la técnica de regularización TSVD. Sabemos que $R$ describe un inverso y un problema mal planteado. Por lo tanto, la matriz de transferencia $A$ está mal acondicionada, por lo tanto, nuestro objetivo es derivar un nuevo problema con una matriz de transferencia bien acondicionada. Para obtener una matriz de transferencia bien acondicionada, necesitamos omitir los valores singulares de $A$ que contienen información relacionada con el ruido y los errores. Una matriz deficiente en rango, que no contenga esta información, se puede obtener una SVD de la matriz de transferencia $A$, es decir,

$$
A_{w}(y, x)=\sum_{n=1}^{w} \sigma_{n} \sigma_{n} \sigma_{n}, \quad w \leq N .
$$

Cuando $u_{n}$ y $v_{n}$ son los vectores $n$ de un SVD de $A$, y $n$ son los valores singulares correspondientes. El rango completo de la matriz es $N$ y el tamaño de $w>$ 0 determina el nivel de regularización. La solución $g$ de TSVD se puede calcular mediante la minimización

$$
|| A_{w} g-d \| \text {. }
$$

Sabemos que la solución de este problema de minimización está dada por la seudo inversa de $A_{w}$ :

$$
A_{w}^{\dagger}=\left(A_{w}^{T} A_{w}\right)^{-1} A_{w}^{T} .
$$

Escribimos $A_{w}^{\dagger}$ en términos de una SVD por 


$$
A_{w}^{\dagger}=\tilde{V} \tilde{\Sigma}^{-1} \widetilde{U}
$$

con $\widetilde{U}$ y $\widetilde{V}$ que contiene el primer $w$ columnas de $U$ y $V$, respectivamente. $\tilde{\Sigma}$ es a $w \times w$ una submatriz diagonal de $\Sigma$

Para obtener la solución regularizada, calculamos

$$
g=A_{w}^{\dagger}(d)
$$

Si insertamos la ecuación (17) en (18), encontramos

$$
g=\tilde{V} \tilde{\Sigma}^{-1} \widetilde{U}^{T}(d)=\sum_{n=1}^{w} \frac{\left\langle u_{n}, d\right\rangle}{\sigma_{n}} u_{n},
$$

donde $\langle\because$,$\rangle denota el producto interno.$

\section{Conclusiones}

En este trabajo se presentó una introducción al problema inverso en electrocardiografía y se describió como se puede resolver mediante el método de regularización de Tikhonov y la TSVD. Al mismo tiempo se mostró el diseño de un sistema de adquisición de señales electrocardiográficas a través de un sistema de adquisición de bajo costo para obtener las 12 derivaciones que en un trabajo futuro permitirán estudiar problemas asociados a algunas cardiopatías y síndromes que se manifiestan en el ventrículo izquierdo. El principal resultado del trabajo es la propuesta de diseño del sistema de captura de bajo costo de señales ECG. En un trabajo futuro se utilizará el sistema propuesto para resolver numéricamente el problema inverso electrocardiográfico que podrá servir para concientizar a la población de algunas de las principales enfermedades cardiacas y así poder ayudar en el entrenamiento médico. Además, se estudiará la estabilidad de ambos métodos de regularización.

\section{Referencias}

1. Gulrajani, R. M.: The forward and inverse problems of electrocardiography. IEEE Engineering in Medicine and Biology Magazine, 17(5), pp. 84-101 (1998)

2. Kochinke, J.: Einfluss von Normierung auf die Analyse von EKG-BSPM Daten (2012)

3. Correa, J. L., Morales, E., Huerta, J. A., González, J. J., Cárdenas, C. R.: Sistema de Adquisición de Señales SEMG para la Detección de Fatiga Muscular. Revista Mexicana de Ingeniería Biomédica, 37(1), pp. 17-27 (2016)

4. Aguirre Botello, M.: Recopilación con datos de INEGI, OMS y SINAIS, México, Principales Causas de Mortalidad 1938-2014. Recuperado de: http://www.mexicomaxico.org/Voto/MortalidadCausas.htm

5. Brooks, D. H., Ahmad, G., MacLeod, R. S.: Multiply constrained inverse electrocardiography: combining temporal, multiple spatial, and interative regularization. In: Proc 16th Ann IntConf IEEE Eng Med Biol Soc, EEE Press, NewYork, pp 137-138 (1994) 
6. Goldman, L.: Approach to the patient with possible cardiovascular disease. In: Goldman L, Schafer A.I., eds., Goldman's Cecil Medicine, 25th ed. Philadelphia, PA: Elsevier Saunders, chap. 51 (2016)

7. Zemzemi, N.: An iterative method for solving the inverse problem in electrocardiography in normal and fibrillation conditions: A simulation Study. In: International Congress on Electrocardiology, ICE, pp. 53-56 (2014)

8. Berrier, K. L., Sorensen, D. C., Khoury, D. S.: Solving the inverse problem of electrocardiography using a Duncan and Horn formulation of the Kalman filter. IEEE Transactions on biomedical engineering, 51(3), pp. 507-515 (2004)

9. Lines, G. T., Cai, X., Nielsen, B. F., Mardal, K. A., Tveito, A.: Computing the Electrical Activity in the Heart. Monographs in Computational Science and Engineering, Springer (2006)

10. Kochinke, J.: Einfluss von Normierung auf die Analyse von EKG-BSPM Daten (2013) 